\title{
Factors affecting bone maturation in Chinese girls aged $4-8$ years with isolated premature thelarche
}

\author{
Huiping Su', Zhe Su*, Lili Pan', Li Wang ${ }^{1}$, Zhongwei Xu', Gang Peng ${ }^{2}$ and Xianglei Li ${ }^{1}$
}

\begin{abstract}
Background: In isolated premature thelarche (IPT) girls, bone age (BA) is considered consistent with chronological age. However, some IPT girls confirmed by gonadotropin-releasing hormone $(\mathrm{GnRH})$ stimulation test could show another trend. We analysed BA and possible potentiating factors in a selected group of girls aged 4-8 years with IPT.
\end{abstract}

Methods: IPT girls confirmed by GnRH stimulation test aged 4-8 years hospitalized from January 2015 to April 2018 at Shenzhen Children's Hospital were included in this retrospective study. They were divided into two groups with advanced BA of 2 years as the cut-off. Body mass index (BMI) and hormone levels were the main outcome measures, and regression analysis was used to identify independent risk factors. IPT girls were divided into subgroups according to the levels of BMI standard deviation score (SDS), insulin-like growth factor-1 (IGF-1) SDS and dehydroepiandrosterone sulfate (DHEAS) SDS for comparisons of advanced BA.

Results: Overall, 423 subjects were included and classified into the advanced BA group (48.7\%, $n=206)$ and control group $(51.3 \%, n=217)$. The advanced BA group had significantly higher BMI SDS, serum DHEAS SDS, IGF-1 SDS, androstenedione and fasting insulin and significantly lower sex hormone binding globulin (all $p<0.001$ ). Serum IGF1 SDS (OR $=1.926, p<0.001)$, BMI SDS (OR $=1.427, p=0.001)$ and DHEAS SDS $(O R=1.131, p=0.005)$ were independent risk factors for significantly advanced BA. In the multiple linear regression model, serum IGF-1 SDS, BMI SDS and DHEAS SDS were the strongest predictors of advanced BA, accounting for $19.3 \%$ of the variance. According to BMl, 423 patients were classified into three groups: normal weight (56.03\%, $n=237)$, overweight $(19.15 \%, n=81)$ and obesity $(24.82 \%, n=105)$. The proportion of advanced BA in obesity group was significantly higher than those of normal weight and overweight groups $\left(X^{2}=18.088, p<0.001\right)$. In the subgroup with normal weight, higher serum IGF-1 SDS $(p=0.009)$ and DHEAS SDS ( $p=0.003)$ affected BA advancement independent of BMI SDS.

Conclusions: Girls with IPT confirmed by GnRH stimulation test aged 4-8 years might have significantly advanced BA. Obesity was highly associated with advanced BA. Age-specific serum IGF-1 SDS and DHEAS SDS were risk factors for BA advancement independent of BMI.

Keywords: Premature thelarche, Bone maturation, Insulin-like growth factor-1, Dehydroepiandrosterone sulfate, Body mass index

\footnotetext{
* Correspondence: Su_zhe@126.com

'Department of Endocrinology, Shenzhen Children's Hospital, No. 7019,

Yitian Road, Futian District, 518038 Shenzhen, Guangdong Province, People's Republic of China

Full list of author information is available at the end of the article
}

(C) The Author(s). 2020 Open Access This article is licensed under a Creative Commons Attribution 4.0 International License, which permits use, sharing, adaptation, distribution and reproduction in any medium or format, as long as you give appropriate credit to the original author(s) and the source, provide a link to the Creative Commons licence, and indicate if changes were made. The images or other third party material in this article are included in the article's Creative Commons licence, unless indicated otherwise in a credit line to the material. If material is not included in the article's Creative Commons licence and your intended use is not permitted by statutory regulation or exceeds the permitted use, you will need to obtain permission directly from the copyright holder. To view a copy of this licence, visit http://creativecommons.org/licenses/by/4.0/ The Creative Commons Public Domain Dedication waiver (http://creativecommons.org/publicdomain/zero/1.0/) applies to the data made available in this article, unless otherwise stated in a credit line to the data. 


\section{Background}

Isolated premature thelarche (IPT) is defined as isolated breast development without the development of other sexual characteristics in girls before 8 years of age. IPT usually presents before the age of 2 , and approximately $83.1 \%$ of patients show spontaneous regression in 2 years, which may be attributable to the so-called minipuberty [1]. IPT presented in an elder age is also considered to be a benign and self-limiting phenomenon that usually not influence the growth or timing of puberty. Generally, bone age (BA) in IPT girls is consistent with chronological age (CA) [2-4].

However, Stanhope and Brook [5] first described a new clinical situation of IPT in 1990. It was characterized by BA advancement and/or growth acceleration and hypothesized that different hormonal changes probably occur. In 1998, Volta et al. [6] reported that 34 (28.6\%) of 119 girls aged 1-8 years with IPT presented with similar characteristics. So, IPT is not always benign and some may have advanced BA. However, the previous studies had small sample size and infant cases were included, without the further analysis of possible factors affecting BA in IPT.

In clinical practice, we also found that some IPT girls aged 4-8 years who need for further investigations had BA advancement of more than 2 years. Oestradiol levels, the most important factor affecting $\mathrm{BA}$, are within the normal prepubertal range in IPT girls [7]. The reason for advanced BA in this group remains unclear.

Therefore, the present study aimed to analyse the characteristics of bone maturation in Chinese girls aged 4-8 years with IPT and the possible potentiating factors for advanced BA in IPT.

\section{Methods}

\section{Study design and participant selection}

Most of girls aged 4-8 years with breast development were followed up in our outpatient clinic. They were potentially hospitalized for gonadotropin-releasing hormone $(\mathrm{GnRH})$ stimulation test when they presented with no breast tissue regression for at least three months and one or more of the following: (1) Progressive breast development: the progression of breast Tanner stage from one to another was less than half a year; (2) Significant BA advancement: BA was greater than 2 years above CA; (3) Linear growth acceleration: height velocity was above the expected value for gender and age and/or above the familial genetic channel. We identified IPT girls who were confirmed by GnRH stimulation test in the Endocrinology Department of Shenzhen Children's Hospital from January 2015 to April 2018.

This was a retrospective case-control study. The cases consisted of IPT girls with significantly advanced BA (BA minus $\mathrm{CA}$ (BA-CA) $\geq 2$ years). The controls were
IPT girls without significantly advanced BA (BA-CA < 2 years).

The inclusion criteria were as follows: (1) Age at onset of the disease was 4-8 years; (2) Patients with isolated breast development; (3) Patients without other secondary sexual characteristics, pigmentation of the nipple and areola, and growth of pubic hair or armpit hair; (4) Peak luteinizing hormone (LH) level was less than $5 \mathrm{IU} /$ $\mathrm{L}$ and the ratio of peak $\mathrm{LH}$ to peak follicle-stimulating hormone (FSH) in the GnRH stimulation test was less than 0.6; (5) Breast development had no other causes, such as peripheral precocious puberty, exogenous estrogen intake, local mammary gland hyperplasia and so on. There had been 480 IPT girls included in the study initially.

Children with history of hormone treatment, history of intaking nutritional tonic and Chinese traditional medicine, other endocrine disease (including growth hormone $(\mathrm{GH})$ deficiency, thyroid dysfunction, adrenocortical dysfunction or hyperactivity), abnormal bone development, congenital dysplasia, premature birth and small for gestational age were excluded. Ultimately, a total of 423 participants were enrolled in the study (Fig. 1).

According to the values of BA-CA, the participants were divided into the advanced $\mathrm{BA}$ group (BA-CA $\geq$ 2 years) and the control group (BA-CA $<2$ years). According to body mass index (BMI) [8], the participants were classified into three groups: normal weight (BMI $P_{5} \sim P_{85}$ or BMI standard deviation score (SDS) -1.65 to $<1.04$ ), overweight (BMI $P_{85} \sim P_{95}$ or BMI SDS 1.04 to $<$ 1.65 ) and obesity (BMI $\geq P_{95}$ or BMI SDS $\geq 1.65$ ). IPT girls with normal weight were then subdivided into two groups separately according to the levels of serum insulin-like growth factor-1 (IGF-1) SDS and dehydroepiandrosterone sulfate (DHEAS) SDS.

Height and weight were measured, and BMI was calculated as body weight $(\mathrm{kg}) /$ height $\left(\mathrm{m}^{2}\right)$. These values were expressed as age- and sex-specific SDS based on national survey data in 2005 [8, 9]. Breast development was staged according to the Tanner criteria. If the Tanner stages of bilateral breast development were different, the mature side was recorded. Hypothalamicpituitary-gonadal axis function was evaluated by basal sexual hormone levels and the GnRH stimulation test (gonadorelin injections at $2.5-3.0 \mu \mathrm{g} / \mathrm{kg}$, with no more than $100 \mu \mathrm{g}$ administered at a time). Other hormones, including oestradiol, DHEAS, androsterone, sex hormone binding globulin (SHBG), IGF-1 and insulin, were evaluated by enzyme-enhanced chemiluminescence assays (Siemens IMMULITE 2000, Munich, Germany). IGF-1 and DHEAS levels were standardized according to reference data for different ages in China [10, 11]. Lipids, including triglycerides, total cholesterol, high-density 


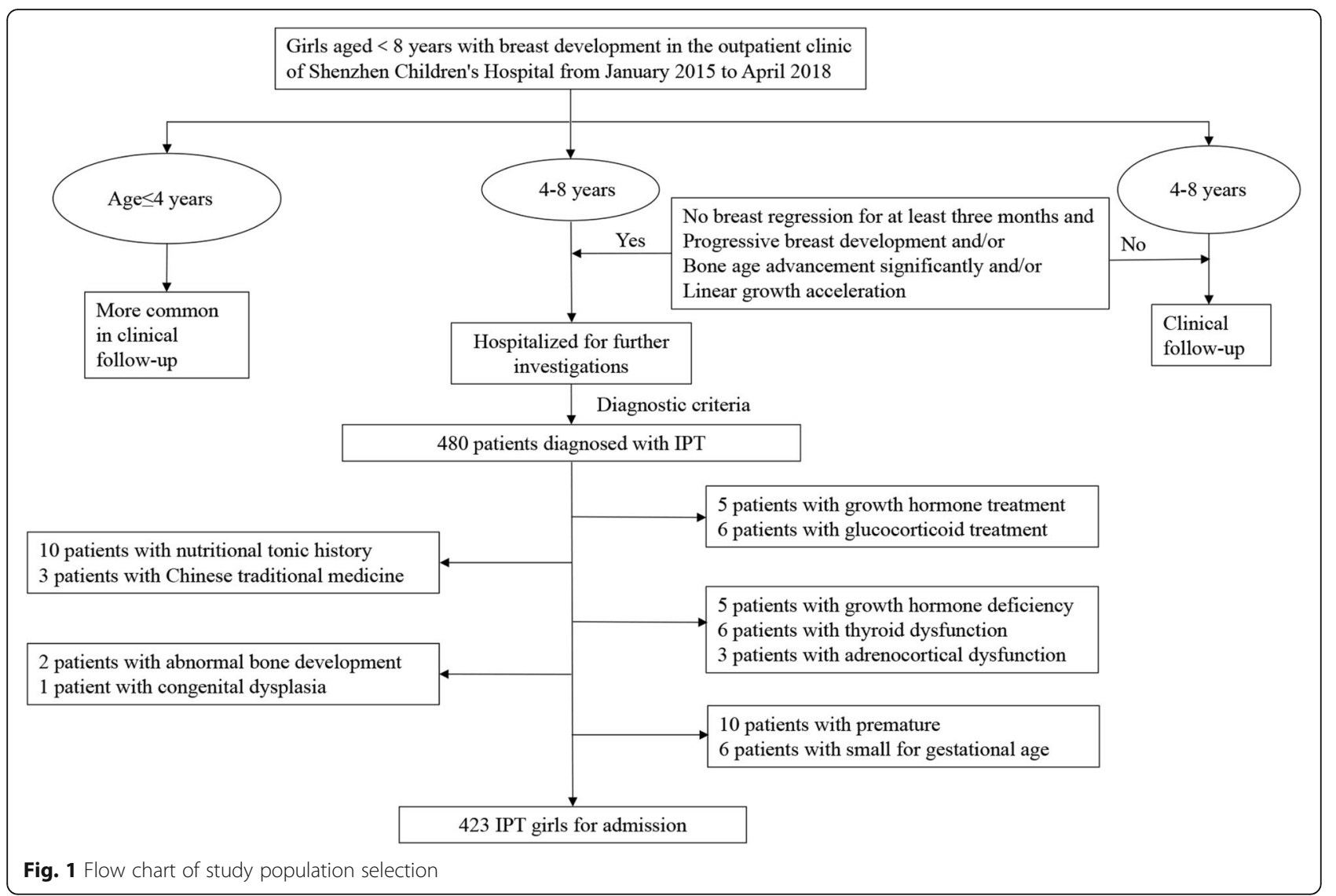

lipoprotein and low-density lipoprotein, were measured using enzymatic end-point method.

We used automated artificial intelligence system (Yitu Healthcare, Hangzhou, China) to evaluate BA on a radiograph of the left hand. This product was version 3 of the Intelligent Diagnosis System for Child Growth and Development, which utilized RUS-CHN. All participants underwent a pelvic ultrasound examination (GE Logic E9 ultrasound machine with a 3.5-12 MHz convex probe and a 9-12 MHz linear probe).

\section{Statistical analysis}

The t-test was used to compare between two groups for the following continuous measures: height (SDS), weight (SDS), BMI (SDS), and BA-CA. Data regarding age at onset, $\mathrm{CA}$, disease duration, uterine length, ovarian volume, basic and peak LH, peak LH/peak FSH, DHEAS (SDS), IGF-1 (SDS), androsterone, insulin, SHBG, oestradiol and lipids were clearly skewed, so median and quartiles were used for these numerical variables. Those differences between two groups were compared using the Mann-Whitney $U$ test. The association between significant BA advancement and breast Tanner stage as well as status of obesity or overweight were calculated using the chi-square test.

In order to identify independent risk factors affecting significantly advanced BA, we performed a binary logistic regression analysis (forward LR stepwise method) with the advanced BA of more than 2 years above CA (yes/no) as the dependent variable. The independent variables were those shown to be significant $(p<0.05)$ in the comparison between two groups (disease duration, BMI SDS, DHEAS SDS, IGF-1 SDS, androsterone, insulin, SHBG, uterine length), significant factors in previous studies (oestradiol), and other factors that may be clinically correlated (basic LH, peak $\mathrm{LH}$, ratio of peak $\mathrm{LH}$ to peak $\mathrm{FSH}$, ovarian volume).

A multiple linear regression model was then used to predict the factors most likely to advance BA, with BA$\mathrm{CA}$ as the dependent variable. After testing the assumptions, we entered the factors shown to be significant $(p<0.05)$ in the comparison between two groups (BMI SDS, DHEAS SDS, IGF-1 SDS, insulin, SHBG, uterine length) and others that may be clinically correlated (peak $\mathrm{LH}$, ratio of peak $\mathrm{LH}$ to peak FSH, ovarian volume) as the independent variables. 
Statistical analyses were performed using SPSS version 23.0. We considered $p$-value $<0.05$ as statistically significant for all analyses.

\section{Results}

\section{General characteristics}

Among the 423 patients, the $\mathrm{BA}$ ranged from 4.60 to 12.50 years, and the BA-CA value, an index of BA advancement, ranged from -0.87 to 4.18 years. Subjects were divided into the advanced $\mathrm{BA}$ group (BA-CA $\geq$ 2 years) and the control group (BA-CA $<2$ years). There were 206 subjects $(48.7 \%)$ in the advanced BA group (BA-CA, $2.63 \pm 0.46$ years) and 217 in the control group (BA-CA, $1.21 \pm 0.59$ years).

\section{Factors influencing BA advancement}

There were no differences in the age of onset, CA at IPT diagnosis or Tanner stage between the two groups (Table 1). Compared to the control group, the advanced BA group had a higher BMI SDS $(p<0.001)$ and a lower height SDS for BA $(p<0.001)$. Serum DHEAS SDS, IGF1 SDS, androstenedione and fasting insulin were significantly higher in the advanced BA group than those in the control group (all $p<0.001$ ). However, SHBG was lower in the advanced BA group $(p<0.001)$. The uterine diameter was longer in the advanced BA group ( $p=$ $0.009)$. The biochemical and imaging characteristics of the study groups are described in Table 2.

\section{Independent risk factors affecting significantly advanced} BA

The results of binary logistic regression analysis (forward LR stepwise method) were presented in Table 3. Serum IGF-1 SDS (OR = 1.926, $p<0.001)$, BMI SDS (OR = 1.427, $p=0.001)$ and DHEAS SDS $(\mathrm{OR}=1.131, p=0.005)$ were independent risk factors for significantly advanced BA. For each 1 SD increase in IGF-1 SDS, BMI SDS and DHEAS SDS above normal, the risk of significantly advanced BA increased by $92.6 \%, 42.7 \%$ and $13.1 \%$, respectively.

\section{A model for predicting BA-CA}

A multiple linear regression model was generated to predict BA-CA. The regression model was statistically significant (Table 4): $F=10.425, p<0.001$. The model could be expressed as presented in equation: Y(BACA $)=1.708+0.168 \times($ IGF-1 SDS $)+0.131 \times($ BMI SDS $)+$ $0.049 \times$ (DHEAS SDS). Serum IGF-1 SDS was the top predictor of BA-CA, followed by BMI SDS and DHEAS SDS. This model accounted for $19.3 \%$ of the variance in BA-CA.

\section{Comparisons of advanced BA in the subgroup}

According to BMI, 423 patients were classified into three groups: normal weight $(56.03 \%, \mathrm{n}=237)$, overweight $(19.15 \%, \mathrm{n}=81)$ and obesity $(24.82 \%, \mathrm{n}=105)$. The proportions of BA advancement in the three groups were $42.6 \%, 43.2 \%$ and $66.7 \%$, respectively. The difference was

Table 1 Physical characteristics of the study population

\begin{tabular}{lccc}
\hline & Advanced BA group & Control group & $p$-value \\
\hline Number of girls & $206(48.7 \%)$ & $217(51.3 \%)$ & - \\
Chronological age (years) & $7.56(6.90,7.95)$ & $7.72(7.06,8.04)$ & 0.189 \\
Disease duration (years) & $0.50(0.25,1.00)$ & $0.33(0.25,0.75)$ & 0.029 \\
Age at onset (years) & $6.86(6.12,7.41)$ & $7.13(6.37,7.60)$ & 0.082 \\
Height-for-age (cm) & $130.77 \pm 6.28$ & $126.76 \pm 7.14$ & $<0.001$ \\
Height-for-age SDS & $1.16 \pm 0.86$ & $0.36 \pm 0.88$ & $<0.001$ \\
Height-for-BA SDS & $-1.45 \pm 0.72$ & $-0.79 \pm 0.95$ & $<0.001$ \\
Genetic target height SDS & $-0.48 \pm 0.80$ & $-0.64 \pm 1.39$ & 0.165 \\
HtBA SDS - THt SDS & $-0.97 \pm 0.80$ & $-0.16 \pm 1.42$ & $<0.001$ \\
Weight-for-age (kg) & $30.06 \pm 5.90$ & $26.58 \pm 4.93$ & $<0.001$ \\
Weight-for-age SDS & $1.46 \pm 1.05$ & $0.64 \pm 0.96$ & $<0.001$ \\
BMl & $17.45 \pm 2.39$ & $16.44 \pm 2.06$ & $<0.001$ \\
BMl SDS & $1.16 \pm 1.10$ & $0.64 \pm 1.07$ & $<0.001$ \\
Breast Tanner stage & & $161(38.1 \%)$ & 0.083 \\
2 & $137(32.4 \%)$ & $56(13.2 \%)$ & $3.24 \pm 0.42$ \\
3 & $69(16.3 \%)$ & $3.25 \pm 0.40$ &
\end{tabular}

Categorical variables are presented as number (\%, percentage). Continuous variables with normal distribution are presented as the mean \pm standard deviation, and variables with non-normal distribution are presented as the median and quartiles

BA Bone age, SDS Standard deviation score, HtBA SDS THt SDS height-for-BA SDS minus genetic target height SDS, BMI Body mass index 
Table 2 Clinical characteristics of the study population

\begin{tabular}{|c|c|c|c|}
\hline & Advanced BA group & Control group & $p$-value \\
\hline Basic LH (IU/L) & $0.24(0.15,0.42)$ & $0.24(0.13,0.44)$ & 0.902 \\
\hline Peak LH (IU/L) & $3.49(2.52,4.18)$ & $3.57(2.80,4.16)$ & 0.301 \\
\hline Peak LH/peak FSH & $0.22(0.17,0.29)$ & $0.21(0.16,0.29)$ & 0.316 \\
\hline DHEAS $(\mu \mathrm{g} / \mathrm{dL})$ & $42.85(23.78,60.83)$ & $29.50(16.80,52.40)$ & $<0.001$ \\
\hline DHEAS SDS & $1.94(0.46,4.15)$ & $0.85(-0.14,2.47)$ & $<0.001$ \\
\hline Androsterone (ng/mL) & $0.30(0.15,0.48)$ & $0.15(0.15,0.35)$ & $<0.001$ \\
\hline Insulin ( $\mu \mid \mathrm{U} / \mathrm{mL})$ & $6.68(5.08,9.37)$ & $5.50(3.56,8.10)$ & $<0.001$ \\
\hline SHBG (nmol/L) & $58.15(41.75,75.23)$ & $71.70(52.80,89.90)$ & $<0.001$ \\
\hline IGF-1 (ng/mL) & $238.00(190.00,300.25)$ & $204.00(168.00,241.00)$ & $<0.001$ \\
\hline IGF-1 SDS & $0.44(-0.30,1.29)$ & $-0.27(-0.73,0.48)$ & $<0.001$ \\
\hline Oestradiol (pmol/L) & $10.00(10.00,20.00)$ & $10.00(10.00,10.00)$ & 0.257 \\
\hline Triglyceride (mmol/L) & $0.80(0.61,1.04)$ & $0.85(0.63,1.05)$ & 0.366 \\
\hline Total cholesterol (mmol/L) & $3.76(3.37,4.35)$ & $3.86(3.49,4.34)$ & 0.299 \\
\hline $\mathrm{HDL}(\mathrm{mmol} / \mathrm{L})$ & $1.23(1.06,1.38)$ & $1.23(1.05,1.42)$ & 0.646 \\
\hline $\mathrm{LDL}(\mathrm{mmol} / \mathrm{L})$ & $2.27(1.96,2.63)$ & $2.36(2.07,2.69)$ & 0.215 \\
\hline Uterine length (cm) & $3.41(3.08,3.67)$ & $3.27(2.93,3.49)$ & 0.009 \\
\hline Ovarian volume (ml) & $1.23(0.82,1.74)$ & $1.13(0.78,1.76)$ & 0.389 \\
\hline
\end{tabular}

Data presented as the median and quartiles

$B A$ Bone age, $L H$ Luteinizing hormone, FSH Follicle-stimulating hormone, DHEAS Dehydroepiandrosterone sulfate, SDS Standard deviation score, SHBG Sex hormone binding globulin, IGF-1 Insulin-like growth factor-1, HDL High-density lipoprotein, LDL Low-density lipoprotein

statistically significant $\left(\chi^{2}=18.055, p<0.001\right)$. It indicated that IPT girls in the obese group had a higher risk of significantly advanced BA. While excluding the influence of obesity/overweight, a high proportion of IPT girls with normal weight still had significantly advanced BA.

In IPT girls with normal weight, we compared the differences of IPT girls with higher serum IGF-1 (IGF-1 $\mathrm{SDS} \geq 0 \mathrm{SD}$ ) and lower IGF-1 (IGF-1 SDS $<0 \mathrm{SD}$ ), and compared those with higher serum DHEAS for age (DHEAS SDS $\geq 2 \mathrm{SD}$ ) to those with normal (DHEAS SDS $<2 \mathrm{SD}$ ). As is shown in Tables 5 and 6, there was no significant difference in BMI SDS between the two groups. After excluding interference by obesity, higher IGF-1 SDS $(p=0.009)$ and DHEAS SDS $(p=0.003)$ independently affect BA advancement.

\section{Discussion}

In this study, nearly half of the IPT girls confirmed by GnRH stimulation test aged 4-8 years had significantly

Table 3 Independent risk factors affecting significantly advanced BA

\begin{tabular}{lllr}
\hline & OR & $95 \% \mathrm{Cl}$ & $p$-value \\
\hline IGF-1 SDS & 1.926 & $1.524-2.436$ & $<0.001$ \\
DHEAS SDS & 1.131 & $1.039-1.232$ & 0.005 \\
BMI SDS & 1.427 & $1.160-1.755$ & 0.001 \\
\hline
\end{tabular}

OR Odds ratio, $\mathrm{Cl}$ Confidence interval, SDS Standard deviation score, IGF-1 Insulin-like growth factor-1, DHEAS Dehydroepiandrosterone sulfate, $B M I$ Body mass index advanced BA, resulting in impaired height for BA. The high proportion of IPT girls with advanced BA in our study may be related with the criteria for requiring further investigations. In addition, one inclusion criterion was age at onset of 4-8 years, which excluded interference by mini-puberty and may increase the probability of observing advanced BA. In contrast to our study, previous studies on IPT have included patients in two categories: patients aged 1-4 years with IPT or all patients younger than 8 years with IPT.

IPT is typically seen in girls with normal growth and normal bone maturation [12]. Oppositely, BA of patients with central precocious puberty (CPP) is generally more advanced than their CA. However, the degree of BA advancement cannot be synchronized with the process of puberty [13]. Patients with CPP accompanied by short disease course or slow pubertal process may not have obvious BA advancement. Thus, the IPT girls with BA advancement should be identified and deserved of more attention.

Advanced BA is found to be influenced by a number of factors in many researches, such as oestrogens [13], androgens [14], GH [15], thyroid hormone [16], nutrition [17], genetics [18] and so on. These studies, however, were mainly aimed at children with obesity. Our study focused on IPT girls and showed that IGF-1, DHEAS and BMI were the most important factors affecting BA advancement, especially the first two independent of BMI. 
Table 4 A model for predicting BA-CA

\begin{tabular}{|c|c|c|c|c|c|c|}
\hline & Beta & Standard error & VIF & $t$ & $p$-value & $\mathrm{R} 2$ \\
\hline (Constant) & 1.708 & 0.386 & & 4.431 & $<0.001$ & \\
\hline IGF-1 SDS & 0.168 & 0.041 & 1.171 & 4.119 & $<0.001$ & \\
\hline DHEAS SDS & 0.049 & 0.015 & 1.141 & 3.241 & 0.001 & \\
\hline BMI SDS & 0.131 & 0.045 & 1.521 & 2.924 & 0.004 & \\
\hline Model & \multicolumn{4}{|c|}{$Y(B A-C A)=1.708+0.168 \times(I G F-1$ SDS $)+0.131 \times(B M I S D S)+0.049 \times($ DHEAS SDS $)$} & $<0.001$ & 19.3 \\
\hline
\end{tabular}

IGF-1 Insulin-like growth factor-1, SDS Standard deviation score, BMI Body mass index, DHEAS Dehydroepiandrosterone sulfate

The important finding of the present study is that higher serum IGF-1 SDS independently affects BA advancement in girls with IPT. For each $1 \mathrm{SD}$ increase in IGF-1, the risk of significantly advanced BA increases by 92.6\%. The GH/IGF-1 axis is critically important for regulating bone maturation. Increased $\mathrm{GH}$ secretion augments circulating IGF-1 levels, inducing the proliferation and differentiation of chondrocytes in the epiphyseal growth plate. Reinehr et al. [19] studied 356 children with obesity and postulated that IGF-1 plays a role in advanced skeletal maturation, independent of sex or pubertal stage. Oppositely, other studies showed no such association $[14,20]$.

In addition, excluding the interference of obesity, IGF1 might be elevated before initiation of puberty. A longitudinal study of 526 healthy children aged 6-16 years showed that the elevated level of circulating IGF-1 occurred 2 years earlier than that of LH level [21]. It may be explained by the expression of IGF-1 receptors in GnRH neurons. Yan et al. [22] infused IGF-1 and antagonist of IGF-1 receptors into the third ventricle of ovariectomized female rats. They found that IGF-1 receptors are required for estradiol activation of $\mathrm{GnRH}$ neurons and for robust GnRH release. Thus, elevated serum IGF1 levels in IPT girls may have a predictive effect on BA advancement and initiation of puberty.

Similar to the findings by Kwon et al. [23] and others $[20,24]$, our study showed a positive association between serum DHEAS SDS and advanced BA. This relationship is likely due to the higher adrenal androgen levels in children with obesity; these increased levels are believed to be responsible for the advanced BA. Giving that obesity is also an important factor in BA advancement, we re-analyzed serum DHEAS SDS and advanced BA in

Table 5 Comparison of IGF-1 SDS in normal weight subgroup

\begin{tabular}{llll}
\hline & IGF-1 SDS $<$ OSD & IGF-1 SDS $\geq$ OSD & $p$-value \\
\hline Number of girls & 120 & 110 & - \\
BA-CA (years) & $1.60 \pm 0.83$ & $1.90 \pm 0.93$ & 0.009 \\
BMI SDS & $0.05 \pm 0.68$ & $0.17 \pm 0.66$ & 0.177 \\
\hline
\end{tabular}

Data presented as mean \pm standard deviation

IGF-1 Insulin-like growth factor-1, SDS Standard deviation score, BA-CA Bone age minus chronological age, BMI Body mass index
IPT girls with normal weight and made the regression analyses. The present study showed that the levels of serum DHEAS are positively associated with BA advancement independent of BMI, which indicates that attention should be paid to the levels of DHEAS even among non-obese prepubertal children.

On the other hand, DHEAS is thought to be a marker of adrenal androgen secretion and adrenarche. It is suggested that whether BA advancement of IPT girls with high level of DHEAS is associated with relatively active adrenal function. However, there are no unified diagnostic criteria for adrenarche at present. The clinical features of adrenarche are considered as development of axillary hair, acne, oily skin and hair, and adult body odor, but patients with these distinguishing features were excluded from the present study population. Su et al. [10] reported that among individuals older than 6 years, serum DHEAS levels increased along with pubertal development in individuals of the same sex in the same age group. It was demonstrated that adrenarche and gonadarche might possibly be related to each other. The higher level of DHEAS may be presented with IPT girls. Therefore, DHEAS could theoretically have independent role at bone maturation of IPT children.

As expected, in this cohort of IPT girls, obesity was highly associated with advanced BA. This is in line with previous studies. Klein et al. [25] studied 167 children across all pubertal stages and from normal weight to obesity and reported that almost $25 \%$ of the obese children had BA advancement by more than 2 SD for CA and that $33 \%$ had BA advancement by more than 2 years above CA and up to 6.5 years. Another cross-sectional study of 66 prepubertal children with premature adrenarche and control subjects reached similar conclusions

Table 6 Comparison of DHEAS SDS in normal weight subgroup

\begin{tabular}{llll}
\hline & DHEAS SDS $<2 S D$ & DHEAS SDS $\geq 2 S D$ & $p$-value \\
\hline Number of girls & 161 & 76 & - \\
BA-CA (years) & $1.64 \pm 0.86$ & $2.00 \pm 0.87$ & 0.003 \\
BMI SDS & $0.10 \pm 0.69$ & $0.13 \pm 0.66$ & 0.759 \\
\hline
\end{tabular}

Data presented as mean \pm standard deviation

DHEAS Dehydroepiandrosterone sulfate, SDS Standard deviation score, $B A-C A$ Bone age minus chronological age, $B M I$ Body mass index 
[20]. The above studies included children at different stages of puberty, which may be influenced by the sexual maturation. Our study cohort only included children with IPT, a relatively homogeneous population, to analyze the relationship between obesity and advanced BA again. In addition, obesity is considered one possible cause of IPT [26]. The effect of BMI on thelarche is likely related to fat mass and the resulting increase in leptin secretion, which is known to affect hypothalamic GnRH-secreting neurons [27]. Thus, more children with IPT may be obese, and the risk of advanced BA may be higher in IPT children.

As shown in multiple linear regression model, IGF-1 SDS, BMI SDS and DHEAS SDS could explain 19.3\% of the variance in advanced $\mathrm{BA}$, suggesting that more unknown factors not included in this study might contribute to advanced BA. Similarly, in a study of 66 prepubertal children with obesity and premature adrenarche, an exploratory stepwise regression model showed that weight, oestradiol and DHEAS were the strongest predictors of $\mathrm{BA} / \mathrm{CA}$, accounting for $24 \%$ of the variance [20]. Another study in 101 obese children generated a backward regression analysis model in which sex, DHEAS SDS, and age explained $27 \%$ of the total variance in BA SDS [24]. Therefore, there may be other underlying causes of advanced BA, including genetic causes such as heterozygous mutations in ACAN [28] and extrinsic factors such as exposure to endocrinedisrupting chemicals [29]. Future studies should clarify the roles of these factors in bone maturation.

Some limitations of our study need to be considered. First, blood samples were not collected in the same batch in this retrospective study. Second, there was no regular follow-up of this group of children. A long-term longitudinal design would offer the opportunity to monitor breast development and BA and determine the final adult height. Therefore, further studies are still needed.

\section{Conclusions}

Girls with IPT confirmed by GnRH stimulation test aged 4-8 years might have advanced BA of more than 2 years above CA, resulting in impaired height for BA. Obesity was highly associated with advanced BA. Age-specific serum IGF-1 SDS and DHEAS SDS were risk factors for BA advancement independent of BMI. For each 1 SD increase, the risk increased by $92.6 \%$ and $13.1 \%$, respectively.

\section{Abbreviations}

IPT: Isolated premature thelarche; BA: Bone age; CA: Chronological age; BACA: Bone age minus chronological age; BMI: Body mass index; SDS: Standard deviation score; LH: Luteinizing hormone; FSH: Follicle-stimulating hormone; GnRH: Gonadotropin-releasing hormone; GH: Growth hormone; DHEAS: Dehydroepiandrosterone sulfate; SHBG: Sex hormone binding globulin; IGF-1: Insulin-like growth factor-1; CPP: Central precocious puberty

\section{Acknowledgements}

Not applicable.

\begin{abstract}
Authors' contributions
ZS designed the study. HS, ZX, GP and XL were responsible for the

acquisition of data. HS, LP and LW made contributions to the analysis and interpretation of data. HS wrote the manuscript. ZS oversaw the planning and execution of the data analysis, as well as reviewed and revised the manuscript. All authors read and approved the final manuscript.
\end{abstract}

\section{Funding}

This study was supported by Shenzhen Healthcare Research Project (No. SZLY2018014). The funders were not involved in the study design, data collection, analysis, interpretation, or manuscript preparation.

\section{Availability of data and materials}

The datasets used and analysed during the present study are available from the corresponding author on reasonable request.

\section{Ethics approval and consent to participate}

This study was approved by the Ethics Committee of Shenzhen Children's Hospital (No. N2019056). All study procedures were conducted in accordance with the tenets of the Declaration of Helsinki, and its subsequent amendments.

\section{Consent for publication}

Not applicable.

\section{Competing interests}

The authors declare that they have no competing interests.

\section{Author details}

'Department of Endocrinology, Shenzhen Children's Hospital, No. 7019, Yitian Road, Futian District, 518038 Shenzhen, Guangdong Province, People's Republic of China. ${ }^{2}$ Department of Adolescent Gynecology, Shenzhen Children's Hospital, Shenzhen, China.

Received: 28 March 2020 Accepted: 23 July 2020

Published online: 29 July 2020

\section{References}

1. Bizzarri C, Spadoni GL, Bottaro G, et al. The response to gonadotropin releasing hormone $(\mathrm{GnRH})$ stimulation test does not predict the progression to true precocious puberty in girls with onset of premature thelarche in the first three years of life. J Clin Endocrinol Metab. 2014;99(2):433-9.

2. Kaplowitz P, Bloch C. Evaluation and Referral of Children With Signs of Early Puberty. Pediatrics. 2016;137(1):e20153732.

3. Sømod ME, Vestergaard ET, Kristensen $K$, et al. Increasing incidence of premature thelarche in the Central Region of Denmark - Challenges in differentiating girls less than 7 years of age with premature thelarche from girls with precocious puberty in real-life practice. Int J Pediatr Endocrinol. 2016; 2016:4.

4. Farello G, Altieri C, Cutini M, et al. Review of the Literature on Current Changes in the Timing of Pubertal Development and the Incomplete Forms of Early Puberty. Front Pediatr. 2019;7:147.

5. Stanhope R, Brook CC. Thelarche variant: a new syndrome of precocious sexual maturation? Acta Endocrinol. 1990;123(5):481-6.

6. Volta C, Bernasconi S, Cisternino M, et al. Isolated premature thelarche and thelarche variant: clinical and auxological follow-up of 119 girls. J Endocrinol Invest. 1998;21(3):180-3.

7. Khokhar A, Mojica A. Premature Thelarche. Pediatr Ann. 2018;47(1):e12-e5.

8. $\mathrm{Li} \mathrm{H}, \mathrm{Ji} \mathrm{CY}$, Zong XN. Body mass index growth curves for Chinese children and adolescents aged 0 to18 years. Chin J Pediatr. 2009;47(7):493-8.

9. Li H, Ji CY, Zong XN. Height and weight standardized growth charts for Chinese children and adolescents aged 0 to 18 years. Chin J Pediatr. 2009; 47(7):487-92.

10. Su Zh, Li YH, Du ML, et al. Change of serum dehydroepiandrosterone sulfate (DHEAS) level in children and adolescents. Chin J Lab Med. 2008; 31(12):1358-62. 
11. Xu SS, Pan H, Zhu HJ, et al. Study on normal reference values of serum insulin growth factor-1 and insulin factor binding protein-3 in children and adolescents. J Clin Pediatr. 2009;27(12):1105-10.

12. Eugster EA. Update on Precocious Puberty in Girls. J Pediatr Adolesc Gynecol. 2019;32(5):455-9.

13. Lazar L, Phillip M. Pubertal disorders and bone maturation. Endocrinol Metab Clin North Am. 2012;41(4):805-25.

14. Kim SE, Jang JW, Ahn MB, et al. The association between skeletal maturation and adrenal androgen levels in obese children and adolescents. Ann Pediatr Endocrinol Metab. 2017;22(2):108-14.

15. Collett-Solberg PF, Jorge AAL, Boguszewski MCS, et al. Growth hormone therapy in children; research and practice - A review. Growth Horm IGF Res. 2019:44:20-32

16. Bassett JH, Williams GR. Role of Thyroid Hormones in Skeletal Development and Bone Maintenance. Endocr Rev. 2016:37(2):135-87.

17. Pinhas-Hamiel O, Benary D, Mazor-Aronovich K, et al. Advanced bone age and hyperinsulinemia in overweight and obese children. Endocr Pract. 2014; 20(1):62-7.

18. McCormack SE, Chesi A, Mitchell JA, et al. Relative Skeletal Maturation and Population Ancestry in Nonobese Children and Adolescents. J Bone Miner Res. 2017;32(1):115-24.

19. Reinehr T, de Sousa G, Wabitsch M. Relationships of IGF-I and andrrogens to skeletal maturation in obese children and adolescents. J Pediatr Endocrinol Metab. 2006;19(9):1133-40.

20. Sopher AB, Jean AM, Zwany SK, et al. Bone age advancement in prepubertal children with obesity and premature adrenarche: possible potentiating factors. Obesity (Silver Spring). 2011;19(6):1259-64.

21. Deng JY, Shi YF, Zhang DX. Effect of insulin-like factor-1 on initiation of puberty in children. Chin J Endocrino Metab. 2005;21(4):337-40.

22. Sun Y, Todd BJ, Thornton $\mathrm{K}$, et al. Differential effects of hypothalamic IGF-I on gonadotropin releasing hormone neuronal activation during steroidinduced $\mathrm{LH}$ surges in young and middle-aged female rats. Endocrinology. 2011:152(11):4276-87.

23. Kwon JH, Lee HA, Kim YJ, et al. Effects of Adrenal Androgen Levels on Bone Age Advancement in Prepubertal Children: Using the Ewha Birth and Growth Cohort Study. J Korean Med Sci. 2017;32(6):968-73.

24. de Groot CJ, van den Berg A, Ballieux B, et al. Determinants of advanced bone age in childhood obesity. Horm Res Paediatr. 2017;87(4):254-63.

25. Klein KO, Newfield RS, Hassink SG. Bone maturation along the spectrum from normal weight to obesity: a complex interplay of sex, growth factors and weight gain. J Pediatr Endocrinol Metab. 2016;29(3):311-8.

26. Atay $Z$, Turan $\mathrm{S}$, Guran $\mathrm{T}$, et al. The prevalence and risk factors of premature thelarche and pubarche in 4- to 8-year-old girls. Acta Paediatr. 2012;101(2): e71-5.

27. Hausman GJ, Barb CR. Adipose tissue and the reproductive axis: biological aspects. Endocr Dev. 2010;19:31-44.

28. Nilsson O, Guo MH, Dunbar N, et al. Short stature, accelerated bone maturation, and early growth cessation due to heterozygous aggrecan mutations. J Clin Endocrinol Metab. 2014;99(8):E1510-8.

29. Durmaz E, Erkekoglu P, Asci A, et al. Urinary phthalate metabolite concentrations in girls with premature thelarche. Environ Toxicol Pharmacol. 2018;59:172-81.

\section{Publisher's Note}

Springer Nature remains neutral with regard to jurisdictional claims in published maps and institutional affiliations.

Ready to submit your research? Choose BMC and benefit from:

- fast, convenient online submission

- thorough peer review by experienced researchers in your field

- rapid publication on acceptance

- support for research data, including large and complex data types

- gold Open Access which fosters wider collaboration and increased citations

- maximum visibility for your research: over $100 \mathrm{M}$ website views per year

At BMC, research is always in progress.

Learn more biomedcentral.com/submissions 